\title{
Correction to: Cardiovascular, muscular, and skeletal adaptations to recreational team handball training: a randomized controlled trial with young adult untrained men
}

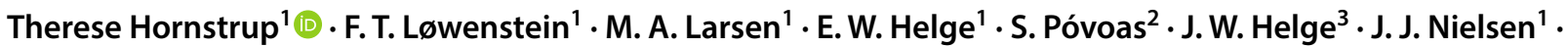 \\ B. Fristrup ${ }^{1} \cdot$ J. L. Andersen ${ }^{4} \cdot$ L. Gliemann $^{1} \cdot$ L. Nybo ${ }^{1} \cdot$ P. Krustrup ${ }^{5,6}$
}

Published online: 13 December 2018

○) Springer-Verlag GmbH Germany, part of Springer Nature 2018

\section{Correction to: European Journal of Applied Physiology https://doi.org/10.1007/s00421-018-4034-5}

The author would like to correct the errors in the publication of the original article. The corrected details are given below for your reading.

In the section "Body composition and bone mineral density", the second sentence should read as:

Proximal femur BMD was calculated as follows: (right $\mathrm{BMD}+$ left $\mathrm{BMD}) / 2$. According to the manufacturer, the coefficient of variation for BMD assessment is $1.0 \%$. In addition, body height and body mass were measured before the DXA scans.
The original article can be found online at https://doi.org/10.1007/ s00421-018-4034-5.

Therese Hornstrup

thornstrup@nexs.ku.dk

1 Department of Nutrition, Exercise and Sports, University of Copenhagen, 2200 Copenhagen N, Denmark

2 Research Center in Sports Sciences, Health Sciences and Human Development, CIDESD, University Institute of Maia, ISMAI, 4475-690 Maia, Portugal

3 Center for Healthy Aging, Department of Biomedical Sciences, University of Copenhagen, 2200 Copenhagen N, Denmark

4 Institute of Sports Medicine Copenhagen, Bispebjerg Hospital, 2400 Copenhagen NV, Denmark

5 Department of Sports Science and Clinical Biomechanics, SDU Sport and Health Sciences Cluster (SHSC), University of Southern Denmark, 3450 Odense, Denmark

6 Sport and Health Sciences, Faculty of Life and Environmental Sciences, University of Exeter, Exeter EX1 2LU, UK
In Figs. 1, 2 and 5, there is red text. It should be written in black. The revised figure (Figs. 1, 2, 5) is placed in the following page. 


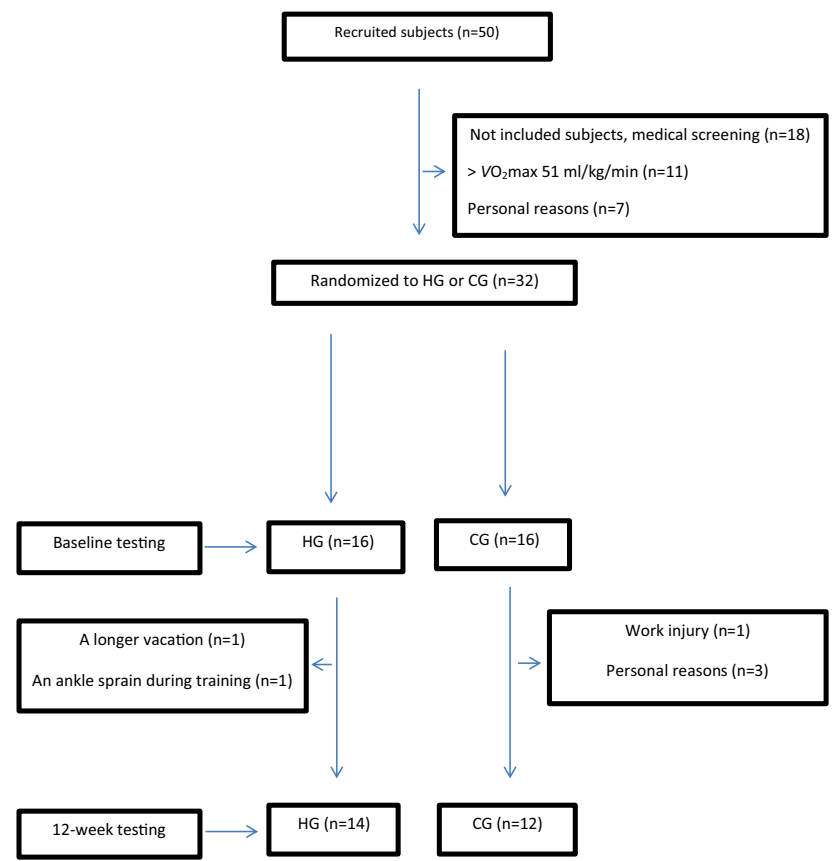

Fig. 1 Flow chart showing the process for recruiting the untrained healthy 20-30-year-old male subjects
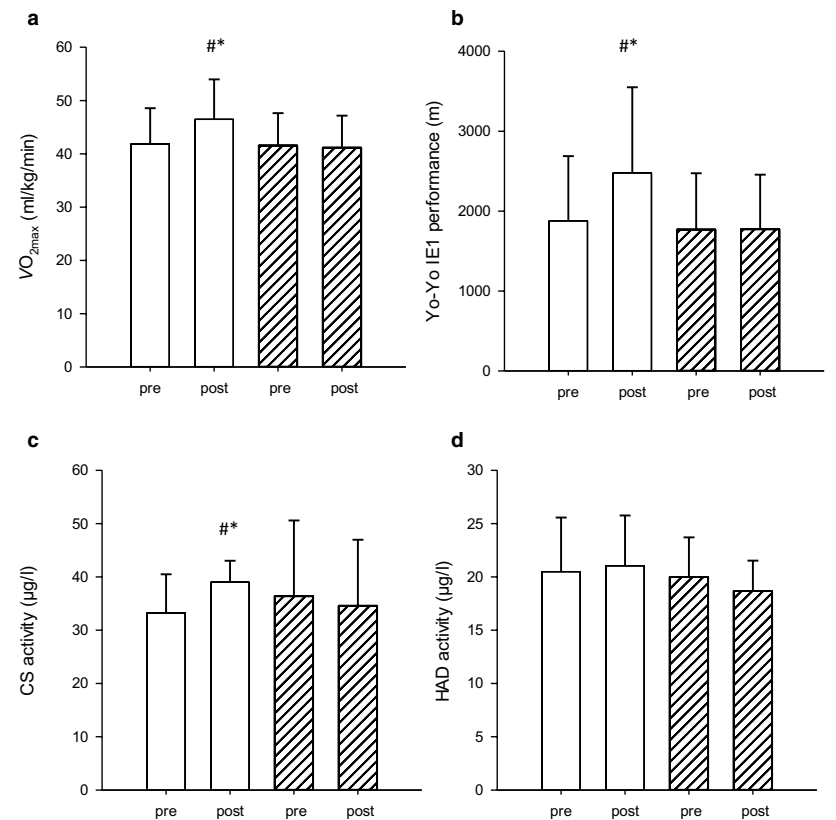

Fig. $2 V_{2 \max }$, Yo-Yo IE1 performance, CS muscle activity, and HAD muscle activity before and after 12 weeks of small-sided handball training (HG, $n=14$ : open bars) and continuation of an inactive lifestyle (CG, $n=12$ : hatched bars). Means $\pm \mathrm{SD}$ are presented. ${ }^{\text {SSig- }}$ nificant $(p<0.05)$ time-by-group interaction in favour of HG. *Significantly $(p<0.05)$ different from 0 week
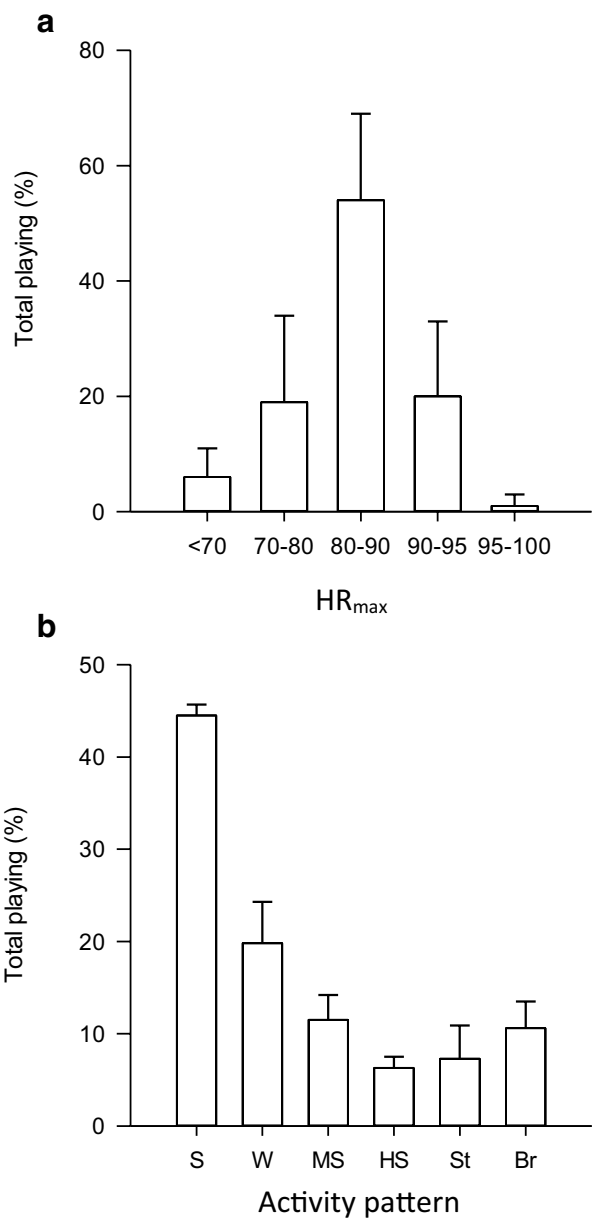

C

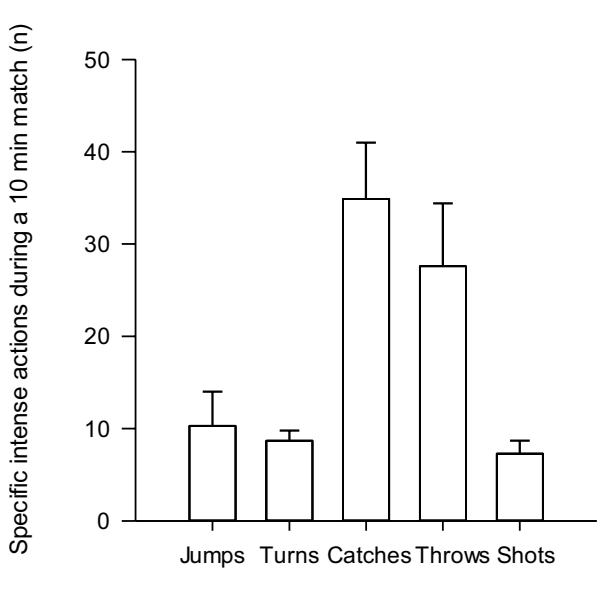

Actions

Fig. 5 Heart-rate distribution expressed in percentage of total playing time (a); activity pattern in various locomotor modes of percentage of total playing time (b): walking (W), standing (S), moderate-speed running (HS), high-speed running (HS), sidesteps (St), and backwards running $(\mathrm{Br})$; number of actions in an 10 min training session (c): jumps, turns, catches, throws, and shots. Means \pm SD are presented 